\title{
Comparison of Norepinephrine and Cafedrine/Theodrenaline Regimens for Maintaining Maternal Blood Pressure during Spinal Anaesthesia for Caesarean Section
}

\author{
Hoyme, M. ${ }^{1}$, Scheungraber, $C^{2}{ }^{2}$, Reinhart, $\mathrm{K}^{3}{ }^{3}$ and Schummer, $\mathrm{W} .{ }^{3}$ \\ ${ }^{1}$ Department of Internal Medicine I (Cardiology, Angiology, Pneumology) Friedrich Schiller University, \\ Jena, Germany \\ ${ }^{2}$ Departments of Obstetrics and Gynecology, Friedrich Schiller University, Jena, Germany \\ ${ }^{3}$ Clinic of Anaesthesiology and Intensive Care Medicine, Friedrich Schiller University, Jena, Germany \\ Correspondence should be addressed to: Schummer, W.; cwsm.schummer@gmx.de
}

Received date: 9 January 2014; Accepted date: 4 April 2014; Published date: 26 August 2015

Academic Editor: Dan Benhamou

Copyright (C) 2015. Hoyme, M., Scheungraber, C., Reinhart, K. and Schummer, W. Distributed under Creative Commons CC-BY 4.0

\begin{abstract}
Common phenomena of spinal anaesthesia for caesarean sections are hypotension and cardiovascular depression, both of which require immediate action. Akrinor ${ }^{\circledR}$ (theodrenaline and cafedrine), a sympathomimetic agent commonly used in Germany for such cases, was phased out with little notice at the end of 2005. Phenylephrine was not and is not an approved drug. Norepinephrine became the first-line drug. The outcome in neonates delivered by elective caesarean section under spinal anaesthesia was studied. At our university hospital, all elective caesarean sections under spinal anaesthesia from 2005 and 2006 were analysed regarding hypotension and the vasopressor administered. If maternal arterial pressure decreased by more than $20 \%$ of baseline, patients in 2005 received Akrinor $\AA$; patients in 2006 received norepinephrine. Neonatal umbilical blood pH as well as Apgar scores at one, five and ten minutes after delivery were measured. Other than the vasopressor, all patients were treated and all data were collected in an identical manner, per our hospital's Standard Operating Procedures (SOP). There were 251 patients eligible for the study; 123 in 2005 and 128 in 2006. A vasopressor was administered to 153 women for treatment of hypotention, 82 women (group A = Akrinor $®$ ) in 2005 and 71 women (group NE = norephinephrine) in 2006. There were no significant differences between the groups regarding arterial umbilical blood $\mathrm{pH}$ or Apgar scores.

Despite treatment, prolonged hypotension ( $>10$ minutes) occurred in both groups (group A: $\mathrm{n}=10$; group NE: $\mathrm{n}=12$ ). The neonatal arterial blood $\mathrm{pH}$ did not show any significant difference between the two groups. Two patients in group A, none in group NE, suffered from clinically relevant tachycardia ( $>50 \%$ increase of baseline). Our results thus suggest that norepinephrine is a safe vasopressor suitable for the treatment of peripartal hypotension without any risk of neonatal acidosis in elective caesarean section under spinal anaesthesia.
\end{abstract}

Keywords: hypotension; Caesarean section; cafedrine, theodrenaline drug combination; norepinephrine. 


\section{Introduction}

Spinal anaesthesia, rather than general anaesthesia, is the choice of modern anaesthetic practice due to pregnancyassociated physiological changes which cause considerable risks regarding airway management. It is the preferred method for elective caesarean sections and is used in $80-90 \%$ of the cases. Even spinal anaesthesia, however, is not without risks. Hypotension is one of the main risks of spinal anaesthesia in this setting and is reported to occur in $80-90 \%$ of cases.

Caesarean sections normally require an anaesthetic block at T4 level, i.e. an extensive sympathetic block with decreased systemic vascular resistance and decreased venous return. Bearing in mind that the mean arterial pressure is defined as cardiac output time's systemic vascular resistance, one must consider that both of these parameters are influenced negatively. (Sharwood-Smith and Drummond 2009) Blood supply and oxygenation of the foetus are dependent on the uteroplacental blood flow. Since the latter is not auto-regulated, a decrease in maternal blood pressure results in a reduction of uteroplacental blood flow and ultimately in impaired foetal oxygenation and (foetal) acidosis.(Shnider et al. 1993) This is the reason why maternal hypotension cannot be tolerated and must be treated aggressively. A variety of vasopressors have been used for this purpose.

Akrinor $®\left(10^{-1}\right.$ vial $=20 \mathrm{mg}$ cafedrine and $1 \mathrm{mg}$ theodrenaline) acts via beta stimulation and consecutively via cyclic adenosine monophosphate, which leads to a positive inotropic action. As a consequence, the stroke volume increases. Heart rate and systemic vascular resistance are considered to remain unchanged. It was used to treat maternal hypotension during elective caesarean section in Germany for decades. (Clemens et al. 2010) At the end of 2005, we were informed that Akrinor $囚$ would be phased out by January 2006. After considering various alternatives, our choice was norepinephrine, a well- established catecholamine in clinical practice.
We then planned a study to compare Akrinor $\AA$ to norephinephrine. Because Akrinor $\AA$ was no longer available for use after 2005; we were compelled to compare the 2005 Akrinor $\AA$ results to the 2006 norephinephrine results. It is important to state that, other than the vasopressor administered, all patients in both years received identical treatment and all data were collected in an identical manner, per our hospital's SOP. The present study investigates differences in the way Akrinor $®$ and norepinephrine affect umbilical arterial $\mathrm{pH}$, Apgar scores, as well as the severity, frequency or duration of hypotension.

\section{Methods}

After approval of the study protocol by the local ethics committee, we targeted all women with singleton pregnancy who preregistered for elective Caesarean section in the years 2005 or 2006 .

The data are collected and maintained in our de-identified data auditing process at our hospital. Patients with cardiovascular problems, pregnancy-induced hypertension or contraindications to spinal anaesthesia were not included. In 2005 the only vasopressor used was Akrinor $\AA$ (group A); in 2006, only norepinephrine was used (group NE). Patients not in need of a vasopressor were assigned to group A0 in 2005 and to group NE0 in 2006.

All women were treated according to the study protocol; i.e. our hospital's SOP regulating spinal anaesthesia in caesarean sections. Premedication was administered upon the anaesthesiologist's request and consisted of famotidine $20 \mathrm{mg}$ the night before, and sodium citrate $(20-30 \mathrm{ml}, 0.3$ $\mathrm{M})$ on arrival at the operating room (OR). Standard monitoring was in place when the patient arrived at the OR and consisted of non-invasive arterial blood pressure, electrocardiography as well as pulse oximetry. External cardiotocography (CTG) was used to monitor the foetal heart rate. After a five-minute rest, baseline arterial blood pressure and heart rate (HR) were recorded. 
After thorough disinfection of the skin at the puncture site, a local anaesthetic was applied. Then a spinal needle (G25 or G27) was inserted at the $\mathrm{L} 2 / 3$ or $\mathrm{L} 3 / 4$ intervertebral space and a mixture of 2.5 $\mathrm{ml}$ hyperbaric $0.5 \%$ bupivacaine and $0.5 \mathrm{ml}$ diluted morphine $(=100 \mu \mathrm{g})$ was injected intrathecally. Then the patient was placed in a supine position with slightly flexed hips and knees, elevated upper body, and a left lateral tilt of the table to prevent inferior vena cava syndrome. The upper part of the body was held in an elevated position. Volume substitution was not applied in advance of spinal anaesthesia but followed the co-load type.(Dyer et al. 2004) Oxygen was administered to all patients from the beginning of the anesthesia when tolerated by the patient. We insisted on oxygen only in patients with a Sp02 $<95 \%$. The caesarean section was performed after five to seven minutes with a sensory level of optimally T4, minimumly T6.

If systolic arterial blood pressure dropped below $100 \mathrm{mmHg}$ or by $20 \%$ of its baseline value, patients in both years were treated according to protocol with a diluted vasopressor (one vial of Akrinor® diluted to $10 \mathrm{ml}$ ) or norepinephrine (diluted to $1 \mathrm{ml}=5 \mu \mathrm{g})$. In 2005, $1 \mathrm{ml}$ of Akrinor ${ }^{\circledR}$ solution $(20 \mathrm{mg}$ cafedrine and $1 \mathrm{mg}$ theodrenaline) was injected repeatedly until baseline pressure was restored. In 2006, $1 \mathrm{ml}$ of norepinephrine (5 $\mu \mathrm{g})$ was administered until baseline pressure was restored.

According to protocol, HR and blood pressure were measured every minute and any deviations from baseline were treated immediately until the umbilical cord was cut. However, the values were recorded in the anaesthesia chart only every five minutes. A prolonged hypotension was defined as systolic blood pressure $<80 \%$ of baseline for more than 10 minutes, despite treatment. A systolic blood pressure > $120 \%$ of baseline was considered reactive hypertension. An increase of the heart rate $>50 \%$ of baseline was considered reactive tachycardia.

Upon obstetricians' request, methylergometrine $(0.2 \mathrm{mg})$ and oxytocine (10 IU) both diluted to $10 \mathrm{ml}$ normal saline were given intravenously. Arterial blood samples from the umbilical cord were preserved by clamping the same on both sides. A bedside blood gas analysis was performed using point-of-care testing (Radiometer, Copenhagen). An arterial pH value under 7.20 was considered as acidosis. Furthermore, Apgar scores one, five and ten minutes after delivery were determined by the obstetricians. The obstetrician did not know what type or dose of vasopressor, if any, had been administered.

\section{Statistics}

Patients in both years, whether they received a vasopressor or not, were compared with respect to size, weight and age using the Student t-test and the MannWhitney $U$ test (Table 1). The primary outcome parameters of the study were umbilical arterial blood $\mathrm{pH}$ and Apgar scores after one, five and ten minutes. Data were tested for normality using the Kolmogorov test and for homogeneity of between- group variance using the Levene test. We compared data using the Student ttest, and the chi-square test. To obtain secondary outcome parameters, we compared the systolic arterial blood pressure and heart rate from induction to delivery, stating incidence and frequency of hypotension, bradycardia, tachycardia and reactive hypertension. We compared data using one- way ANOVA, ANOVA for repeated measures and the Kruskal- Wallis test, with post hoc comparisons using Tukey's honestly significant difference test and the Mann- Whitney U test. All analyses were performed using the SPSS 19.0.0 for Windows. A P- value below 0.05 was considered significant. 
Table 1: Patient Characteristics, Surgical Times. Values are Mean $\pm \mathrm{SD}$, Group $A=$ Treatment with Cafedrine/Theodrenaline, Group NE= Treatment with Norepinephrine, Group A $0=$ No Treatment with Cafedrine/ Theodrenaline Necessary, Group NE $0=$ No Treatment with Norepinephrine Necessary

\begin{tabular}{|cccccc|}
\hline & Group A & Group NE & & Group A 0 & Group NE 0 \\
& $(\mathrm{n}=82)$ & $(\mathrm{n}=71)$ & & $(\mathrm{n}=20)$ & $(\mathrm{n}=28)$ \\
\hline Age (yr) & $31 \pm 4$ & $30 \pm 6$ & 0.5 & $31 \pm 3$ & $28 \pm 4$ \\
Weight (kg) & $82 \pm 18$ & $80 \pm 13$ & 0.4 & $80 \pm 16$ & $74 \pm 13$ \\
$\begin{array}{c}\text { Height (cm) } \\
\begin{array}{c}\text { Induction to } \\
\text { delivery } \\
\text { time (min) }\end{array}\end{array}$ & $165 \pm 7$ & $166 \pm 8$ & 0.8 & $168 \pm 7$ & $167 \pm 5$ \\
\hline
\end{tabular}

\section{Results}

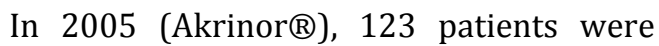
eligible. Due to lack of data (11 patients) and protocol deviation (10 patients), only 102 patients could be studied. In 2006 (norepinephrine), 128 patients were eligible. Due to lack of data (19 patients) and protocol deviations (10 patients), only 99 could be studied. Hypotension did not occur in all of the patients and consequently, not all of the patients required treatment (group $\mathrm{A} 0=20$ patients, group NE0=28 patients).

A total of 153 patients required treatment with a vasopressor. In 2005, 82 women received Akrinorß; in 2006, 71 received norepinephrine (Figure 1). The umbilical arterial $\mathrm{pH}$ values in all four groups were almost identical (Table 2). Three neonates in group A (3.6\%), two in group $\mathrm{NE}$ $(2.81 \%)$ and one in group NE0 (3.5\%) suffered from acidosis (umbilical arterial $\mathrm{pH}<7.20$ ).

There were no significant Apgar score differences between group A and group NE; the two groups in which patients had been treated with a vasopressor (Table 2). Nine neonates in group $\mathrm{A}$ and nine in group $\mathrm{NE}$ had an Apgar score 7 after one minute. After five minutes, two neonates in group A and one in group NE had an Apgar score < 7. After ten minutes, all Apgar scores in both groups were at least 7 . 
Table 2: Neonatal Outcome. Values are Mean or Numbers (Percent).

\begin{tabular}{|c|c|c|c|c|c|c|}
\hline & $\begin{array}{l}\text { Group A } \\
(n=82)\end{array}$ & $\begin{array}{l}\text { Group NE } \\
(n=71)\end{array}$ & $\begin{array}{c}\text { P-value } \\
\text { (treatment } \\
\text { Groups) }\end{array}$ & $\begin{array}{c}\text { Group A } 0 \\
(n=20)\end{array}$ & $\begin{array}{c}\text { Group NE0 } \\
(n=28)\end{array}$ & $\begin{array}{c}\text { P-value } \\
\text { (all groups) }\end{array}$ \\
\hline $\begin{array}{l}\text { Umbilical } \\
\text { arterial blood } \\
\text { gases pH }\end{array}$ & $7.29 \pm 0.05$ & $7.30 \pm 0.06$ & 0.3 & $7.31 \pm 0.05$ & $7.30 \pm 0.04$ & 0.4 \\
\hline $\begin{array}{l}\text { Apgar score at } 1 \\
\min \end{array}$ & 8 & 8 & 0.1 & 8 & 8 & \\
\hline $\begin{array}{c}\text { Apgar score at } 5 \\
\text { min }\end{array}$ & 9 & 9 & 0.1 & 10 & 9 & \\
\hline $\begin{array}{c}\text { Apgar score at } \\
10 \mathrm{~min}\end{array}$ & 9 & 10 & 0.06 & 10 & 9 & \\
\hline $\begin{array}{c}\text { Apgar score }<7 \\
\text { at } 1 \mathrm{~min}\end{array}$ & $9(10.9 \%)$ & $9(12.7 \%)$ & & 3 (15\%) & $1(3.6 \%)$ & \\
\hline $\begin{array}{l}\text { Arterial blood } \\
\mathrm{pH}<7.20\end{array}$ & $3(3.6 \%)$ & $2(2.81 \%)$ & & 0 & $1(3.5 \%)$ & \\
\hline
\end{tabular}

Hoyme, M., Scheungraber, C., Reinhart, K. and Schummer, W (2015), Obstetrics \& Gynecology: An International Journal, DOI: 10.5171/2015.714966 


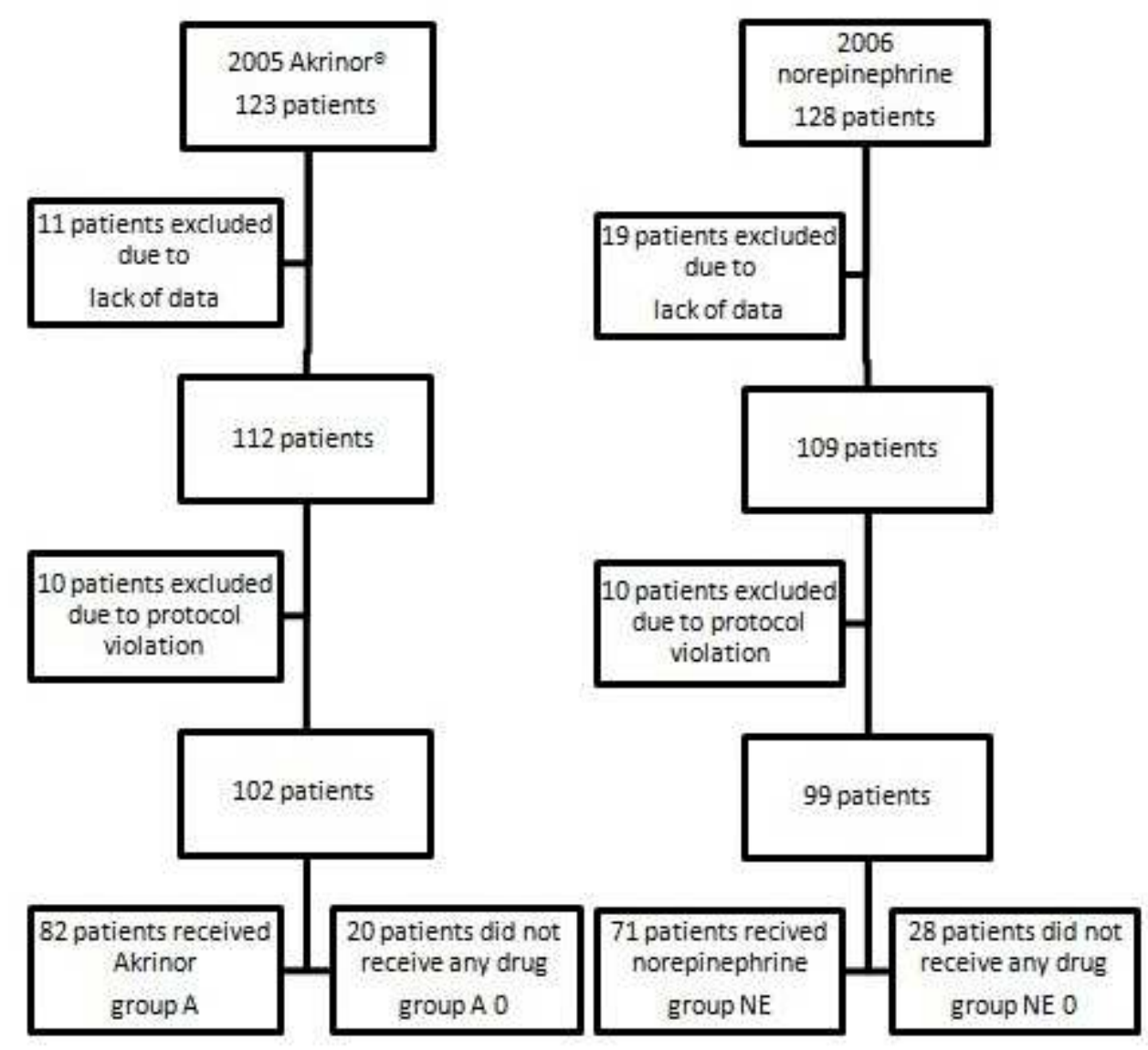

Figure 1: Enrolment

As for the systolic blood pressure, there was a trend towards faster stabilisation under treatment with Akrinor@ during the first five minutes $(96.0 \%$ versus $88.5 \%$; $\mathrm{P}=0.001)$. There were no significant differences between the two groups after ten minutes ( $96.7 \%$ versus $96.3 \%$; $\mathrm{P}=0.88$ ). The course of arterial blood pressure is shown in Figure 2. 


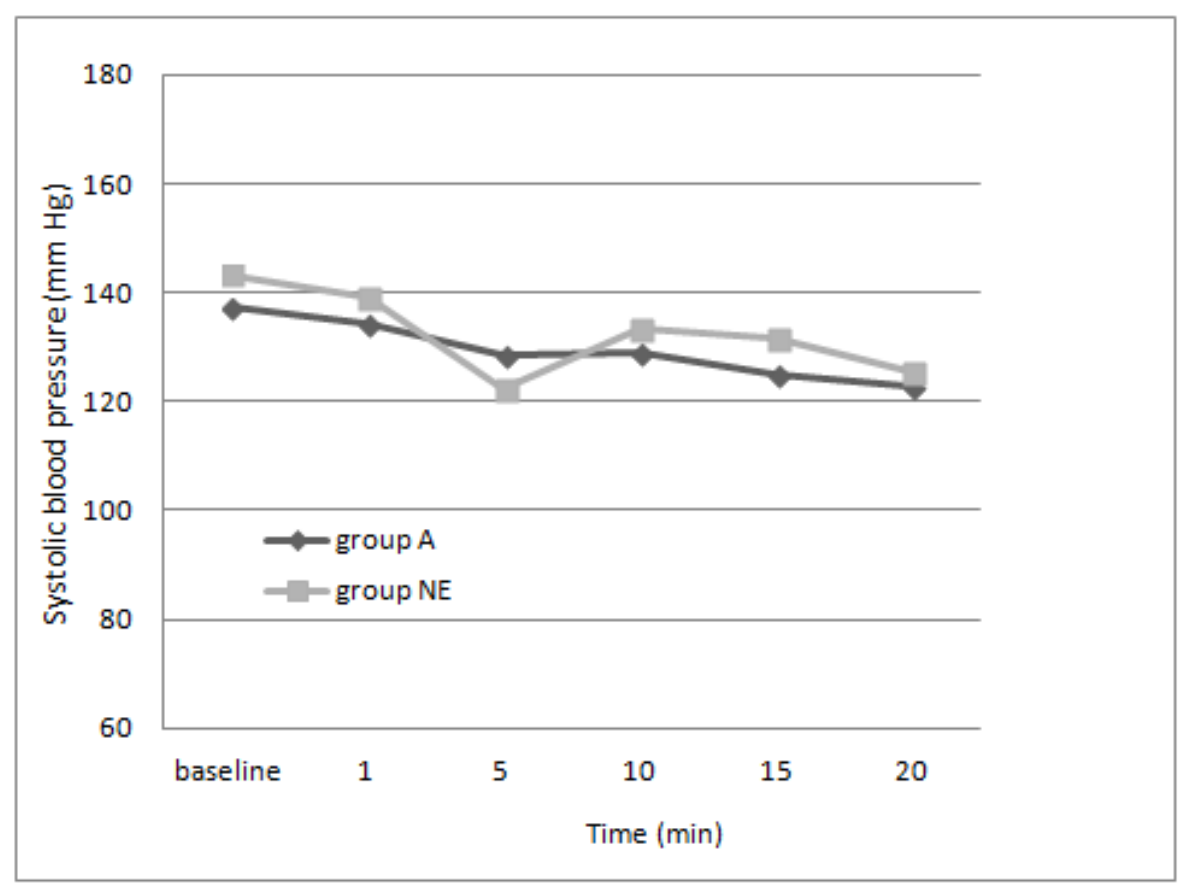

Figure 2: Serial Changes in Systolic Blood Pressure.

\section{Group A= Cafedrine/Theodrenaline, Group NE= Norepinephrine}

Despite administration of a vasopressor, ten women in group $\mathrm{A}$ and 12 in group $\mathrm{NE}$ suffered from prolonged hypotension for more than ten minutes. Three neonates in group A and one in group NE had an Apgar score $<7$ after one minute. There were no statistically significant differences between the groups in arterial blood $\mathrm{pH}$ (group $\mathrm{A}$ : 7.27 versus group NE: $7.29 \mathrm{P}=0.5$ ) and in Apgar scores (Table 3).

Table 3: Neonatal Outcome in Subgroups with Prolonged Hypotension (Systolic Blood Pressure $<80 \%$ of Baseline after 10 Min). Values are Mean \pm SD or Numbers (Percent).

\begin{tabular}{|cccc|}
\hline & Group A & Group NE & P- value \\
\hline $\begin{array}{r}\text { Umbilical arterial blood } \\
\text { gases } \mathrm{pH}\end{array}$ & $7.27 \pm 0.06$ & $7.29 \pm 0.09$ & 0.5 \\
Apgar score $<7$ at 1 min & $3(30 \%)$ & $1(8.3 \%)$ & 0.2 \\
Apgar score $<7$ at 5 min & $2(20 \%)$ & $1(8.3 \%)$ & 0.4 \\
Apgar score $<7$ at 10 min & 0 & 0 & \\
\hline
\end{tabular}


A reactive hypertension (increase of SBP by more than $20 \%$ of baseline value) was recorded in three patients in group $\mathrm{NE}$ and six patients in group $A$.

The course of the heart rate is shown in Figure 3. After five minutes, $\mathrm{HR}$ was proportionally closer to the baseline value in group $\mathrm{A}$ as compared with group NE without being statistically significant (98\% versus $94 \% ; \mathrm{P}=0.06$ ). After ten minutes, HR continued to be statistically insignificant between the two groups $(97.1 \%$ versus 95.4\%; $\mathrm{P}=0.42$ ).

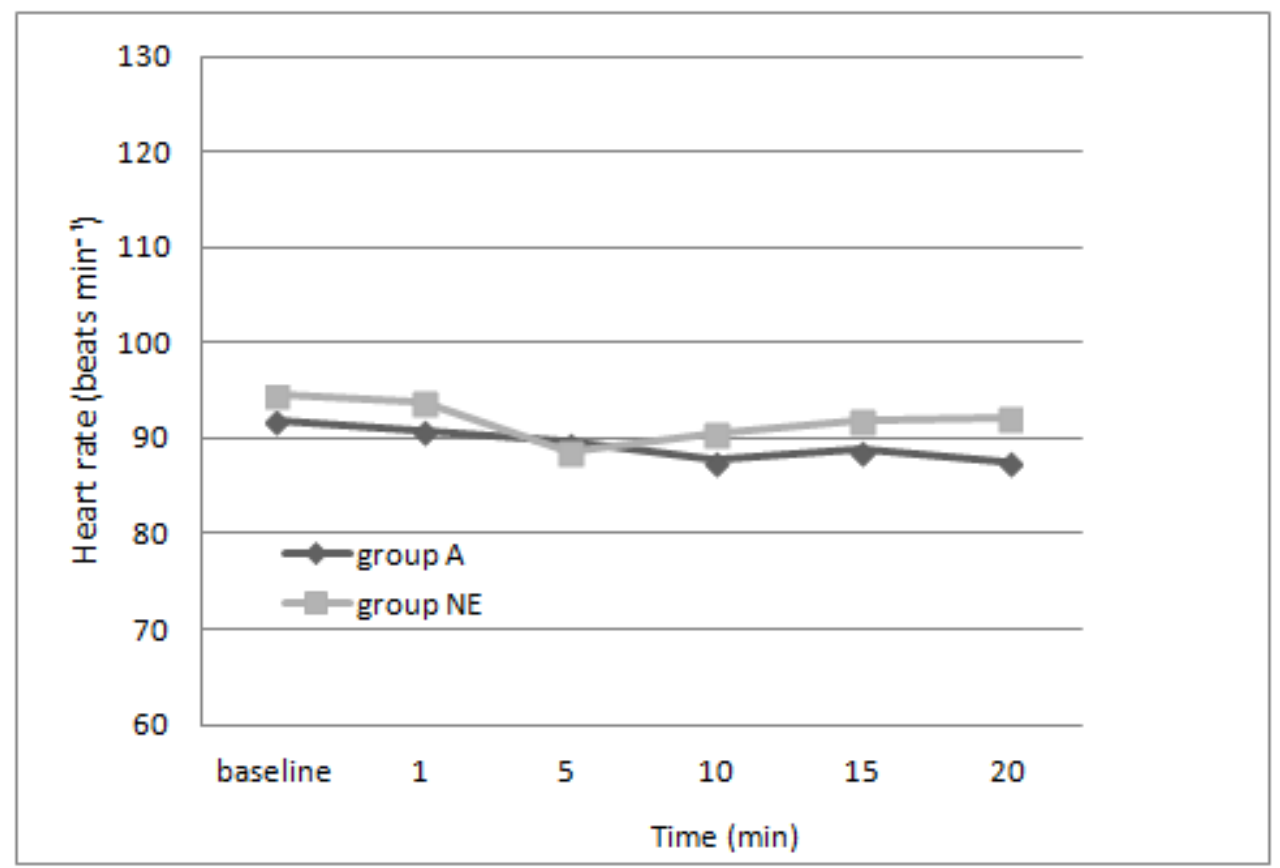

Figure 3: Serial Changes in Heart Rate.

\section{Group A= Cafedrine/Theodrenaline, Group NE= Norepinephrine}

Two patients in group A and none in group NE had reactive tachycardia (increase of HR by $>50 \%$ of baseline value) which was considered clinically relevant and potentially dangerous. None of the patients was bradycardic ( $\mathrm{HR}<50)$. In group A, 21 patients $(25 \%)$ and in group NE, 25 patients (35\%), respectively, suffered from nausea $(\mathrm{P}=0.1)$. Of these patients, two in each group actually vomited (Table 4$)$. 


\section{Table 4: Nausea/Vomiting, Maternal Haemodynamic Changes (Systolic Blood Pressure $<\mathbf{8 0} \%$ of Baseline. Systolic Blood Pressure $>\mathbf{1 2 0 \%}$ of Baseline). Values are Numbers (Percent).}

\begin{tabular}{|c|c|c|c|c|c|}
\hline & $\begin{array}{c}\text { Group A } \\
(n=82)\end{array}$ & $\begin{array}{c}\text { Group NE } \\
(n=71)\end{array}$ & P value & $\begin{array}{c}\text { Group A } 0 \\
(n=20)\end{array}$ & $\begin{array}{c}\text { Group NE } 0 \\
(n=28)\end{array}$ \\
\hline Nausea & $21(25 \%)$ & 25 (35\%) & $\mathrm{P}=0.1$ & $3(15 \%)$ & $1(3 \%)$ \\
\hline Vomiting & $2(2 \%)$ & $2(3 \%)$ & & 0 & 0 \\
\hline $\begin{array}{l}\text { Prolonged } \\
\text { hypotension }\end{array}$ & $10(12 \%)$ & $12(17 \%)$ & $\mathrm{P}=0.2$ & 0 & 0 \\
\hline $\begin{array}{c}\text { Reactive } \\
\text { hypertension }\end{array}$ & $6(7 \%)$ & $3(4 \%)$ & & 0 & 0 \\
\hline Bradycardia & 0 & 0 & & 0 & 0 \\
\hline Tachycardia & $2(2 \%)$ & 0 & & 0 & 0 \\
\hline
\end{tabular}

\section{Discussion}

To date, anaesthesia-associated hypotension has been at the top of the list of perioperative complications during elective caesarean sections and it represents a serious risk for the newborns' health.(Rout and Rocke 1994)

In our study, we cogently demonstrate that treating anaesthesia-associated hypotension with either norepinephrine or Akrinor $®$ produces only insignificant differences in the outcome of neonates, as measured by umbilical blood $\mathrm{pH}$ and Apgar scores. Both drugs are administered to maintain blood flow and neither drug produces acidosis. In fact, because uteroplacental blood flow is not autoregulated, but directly coupled to maternal blood pressure, maternal hypotension must be treated immediately to lessen the risk of foetal acidosis. Given these findings, norepinephrine should be seen as a safe vasopressor suitable for treating women undergoing elective caesarean section.

Traditionally, ephedrine was internationally regarded as the first-choice drug to maintain maternal arterial blood pressure.(Husaini and Russell 1998; Morgan 1994; Turkoz et al. 2002) Ephedrine, by beta 1 stimulation, causes an increase of cardiac output and indirectly effects vasoconstriction through the release of norepinephrine. Repeated administration of ephedrine, however, diminishes the vasoconstrictive effect, since the norepinephrine supply dwindles. Compared to vasopressors, uteroplacental blood flow was less compromised but ephedrine in higher dosages does cross the placenta, leading to an increase in foetal sympathetic tone. Due to ephedrine`s slow onset of action, foetal tachycardia may appear rather unexpectedly. If tachycardia appears in concurrence with a pre-existing oxygen deficit, it may lead to acidosis. 
Therefore, in order to avoid foetal tachycardia, one should avoid ephedrine. In recent years, most likely related to the adverse effects of ephedrine, phenylephrine has become the preferred drug to maintain maternal blood pressure. Phenylephrine acts through alpha-1 stimulation and induces a contraction of vascular smooth muscle cells lasting up to 20 minutes.(Morgan 1994) It gained popularity because of the fast onset and because it is easy to titrate. In contrast to the beta mimetic ephedrine, no acidosis was observed with phenylephrine. However, in larger doses, it decreases uteroplacental perfusion. (Cooper et al. 2002; Prakash et al. 2010)

Since phenylephrine has never been approved in Germany, we began using norepinephrine, which binds to adrenergic alpha 1 , such as phenylephrine, as well as beta 1 receptors. Hence, it increases systolic blood pressure by increasing both peripheral vascular resistance and cardiac output. Simply by the application of norephinephrine, the immediate consequence of the sympathetic block induced by spinal anaesthesia can be antagonized.

From a theoretical point of view, reflex bradycardia through the baroreceptor reflex may be a concern. The vasoconstriction and increase in systolic blood pressure cause an increase of the vagal tone, which leads to a reflex bradycardia. This, however, can be antagonized very well using atropine and its fast vagolytic effect. Our data clearly support this. No patient suffered from an $\mathrm{HR}<50$. Atropine or glycopyrrolate should always be available when alpha agonists are used. Because of its quaternary structure, glycopyrrolate crosses the placenta less than atropine and would, therefore, be the most appropriate anticholinergic agent. Unfortunately, glycopyrrolate has also not been approved for use in Germany.

One of the main concerns in using alpha agonists is a decrease in uteroplacental blood flow. Impairment in foetal oxygenation could be demonstrated in monkeys when epinephrine and norepinephrine were administered to the mother. (Adamsons et al. 1971) In ovine animals treated with ephedrine, an increased endothelial NO expression with subsequent dilation of the uteroplacental circulation seems to preserve uterine blood flow and optimize both foetal oxygenation and acid-base balance.(Li et al. 1996) In humans, Minzter and colleagues tested the diverse effects of vasopressors on placental circulation in a dual perfused human placenta. Here, norepinephrine had no effect on foetal arterial perfusion pressure over 180 minutes when the maternal chorionic plate was perfused with a $250 \mathrm{ml}$

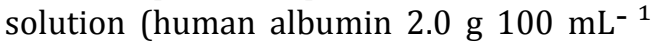
and glucose $150 \mathrm{mg} 100 \mathrm{~mL}^{-1}$ ) containing $5 \mathrm{mg}$ norepinephrine. The hypothesis that alpha-adrenergic agonists compromise the foeto- placental microcirculation could be dismissed.(Minzter et al. 2010) This was also true in our study. All four of our groups recorded insignificant differences in arterial blood pH values, and none of the groups showed any signs of impaired foetal oxygenation.

In contrast with norepinephrine, the vasopressor cafedrine/ theodrenaline (trade name Akrinor $囚$ ) often used in Germany until 2006, does not act via an increase of the systemic vascular resistance but via beta stimulation and consecutively via cyclic adenosine monophosphate, which leads to a positive inotropic and chronotropic action. Like etilefrine and ephedrine, Akrinor $\AA$ also restores the uteroplacental perfusion (in an ovine model). In contrast with ephedrine, however, the restoration of uterine perfusion was delayed and less pronounced.(Strumper et al. 2005) Our data demonstrate a prompt and adequate restoration of systolic blood pressure after hypotension associated with spinal anaesthesia.

The extent and duration of hypotension are regarded as causes of nausea and vomiting. In our study, fewer patients suffered from nausea in the Akrinor $\AA$ group. One explanation may be that the restoration of blood pressure during the first five minutes was faster with the use of Akrinor@. 
The use of Akrinor $\AA$, however, may be harmful due to an overshoot in haemodynamics: Two of the Akrinor®treated patients suffered from tachycardia, and six patients from reactive hypertension.

Regarding the use of norepinephrine in patients with prolonged hypotension, the Apgar scores were significantly higher in this group. In this regard, norepinephrine seems to be superior. We should emphasize, however, that norepinephrine should be given at a very early stage and, if hypotension occurs, in higher doses.

It would be desirable to execute a study comparing norepinephrine with phenylephrine, in order to understand how these two drugs differ in action and whether there is a difference in outcome.

From a theoretical point of view, norepinephrine should be an almost ideal drug to balance haemodynamic compromise of spinal anaesthesia, especially considering the short half-life of this vasopressor.

Admittedly, our study lacks the process of randomising and double blinding; however, there were no differences between groups regarding physical size, weight and age. Our hospital's SOP for spinal anaesthesia for caesarean section dictates that blood pressure and heart rate be monitored continuously, and any deviation from baseline be treated immediately. We can only present data recorded in the charts and must bear in mind that deviations might have been more dramatic. Since the study only included healthy women, the conclusions drawn must be restricted accordingly.

From our data we can only judge that norepinephrine is not inferior to Akrinor in elective caesarean sections under spinal anaesthesia. It would be rather interesting to compare NE with phenylephrine which nowadays is considered the drug of choice to treat maternal hypotension.

In conclusion, we can say that both $\mathrm{NE}$ and Akrinor $\AA$ are suitable and potent drugs to counterbalance the haemodynamic effects of spinal anaesthesia associated with sympathetic block. In healthy women, the outcome is the same for the application of either drug. However, NE, through its pharmacological profile, represents the vasopressor which is associated with lower risks.

\section{Funding}

There was no funding.

\section{Acknowledgements:}

We thank Mrs. Antje Brandstaedt for her help with statistical analysis and Professor Schleussner for his cooperation with this study performed in his department.

Declaration of interests: All authors declare that they have no competing interests relevant to this manuscript.

\section{References}

1.Adamsons, K., E. Mueller-Heubach \& R. E. Myers. (1971). "Production of Fetal Asphyxia in the Rhesus Monkey by Administration of Catecholamines to the Mother," American Journal of Obstetrics Gynecology 109(2):248-262.

2.Clemens, K. E., I. Quednau, A. R. Heller \& E. Klaschik. (2010.) 'Impact of Cafedrine/Theodrenaline (Akrinor(R)) On Therapy of Maternal Hypotension during Spinal Anesthesia for Cesarean Delivery: A Retrospective Study,' Minerva Ginecol 62(6):515-524.

3.Cooper, D. W., M. Carpenter, P. Mowbray, W. R. Desira, D. M. Ryall \& M. S. Kokri. (2002). "Fetal and Maternal Effects of Phenylephrine and Ephedrine during Spinal Anesthesia for Cesarean Delivery," Anesthesiology 97(6):1582-1590.

4.Dyer, R. A., Z. Farina, I. A. Joubert, P. Du Toit, M. Meyer, G. Torr, K. Wells \& M. F. James. (2004). "Crystalloid Preload versus Rapid Crystalloid Administration after Induction of Spinal Anaesthesia (Coload) For Elective Caesarean Section," Anaesth Intensive Care 32(3):351-357. 
5.Husaini, S. W. \& I. F. Russell. (1998). "Volume Preload: Lack of Effect in the Prevention of Spinal-Induced Hypotension at Caesarean Section," International Journal of Obstetric Anesthesia 7(2):76-81.

6.Li, P., C. Tong \& J. C. Eisenach. (1996). "Pregnancy and Ephedrine Increase the Release of Nitric Oxide in Ovine Uterine Arteries," Anesthesia \& Analgesia 82(2):288-293.

7.Minzter, B. H., R. F. Johnson, R. L. Paschall, R. Ramasubramanian, G. D. Ayers \& J. W. Downing. (2010). "The Diverse Effects of Vasopressors on the Fetoplacental Circulation of the Dual Perfused Human Placenta," Anesthesia \& Analgesia.

8.Morgan, P. (1994). "The Role of Vasopressors in the Management of Hypotension Induced by Spinal and Epidural Anaesthesia," Canadian journal of anaesthesia 41(5 Pt 1):404-413.

9.Prakash, S., V. Pramanik, H. Chellani, S. Salhan \& A. R. Gogia. (2010). "Maternal and Neonatal Effects of Bolus Administration of Ephedrine and Phenylephrine during Spinal Anaesthesia for Caesarean Delivery: A Randomised Study," International Journal of Obstetric Anesthesia 19(1):24-30.

10.Rout, C. C. \& D. A. Rocke. (1994). "Prevention of Hypotension Following Spinal Anesthesia for Cesarean Section," In International Anesthesiology Clinics.
11.Sharwood-Smith, G. \& G. B. Drummond. (2009). "Hypotension in Obstetric Spinal Anaesthesia: A Lesson from PreEclampsia," British journal of Anaesthesia 102(3):291-294.

12.Shnider, S., G. Levinson \& E. Cosmi. (1993). 'Obstetric Anesthesia and Uterine Blood Flow,' In Anesthesia for Obstetrics, eds. Shnider S. and Levinson G. 3. Edition. Baltimore: Williams \& Wilkins.

13.Strumper, D., W. Gogarten, M. E. Durieux, K. Hartleb, H. Van Aken \& M. A. Marcus. (2005). "Effects of cafedrine/Theodrenaline, Etilefrine and Ephedrine on Uterine Blood Flow during Epidural-Induced Hypotension in Pregnant Sheep," Fetal Diagnosis and Therapy 20(5):377-382.

14.Turkoz, A., T. Togal, R. Gokdeniz, H. I. Toprak \& O. Ersoy. (2002). 'Effectiveness of Intravenous Ephedrine Infusion during Spinal Anaesthesia for Caesarean Section Based on Maternal Hypotension, Neonatal Acid-Base Status and Lactate Levels,' Anaesthtic Intensive Care 30(3):316-320. 